\title{
Control of the optically induced heating of gold nanoparticles
}

\author{
G. Palermo, R. Caputo, A. De Luca, and C. Umeton \\ Department of Physics and CNR-NANOTEC, University of Calabria, Arcavacata di Rende, 87036 Cosenza, Italy
}

Received February 14, 2017; accepted February 21, 2017; published March 31, 2017

\begin{abstract}
Gold nanoparticles (GNPs) have proven to be good nanosources of heat in the presence of specific electromagnetic radiation. This process, in fact, becomes strongly enhanced under plasmon resonance. In particular, the amount of generated heat and the consequent temperature increase depend on the number of GNPs that are collectively excited and on their relative distance. As a result, the regime of heat localization is deeply controlled by this last parameter.
\end{abstract}

Thermo-plasmonics is one of the most emerging and fastest growing research fields of the recent years. Based on the use of noble metal nanoparticles (NPs) to control temperature at the nanoscale, thermo-plasmonics exploits the localized surface plasmon resonance (LSPR) [1-3], which occurs when the conduction electrons of NPs oscillate in resonance with the electric field of an impinging light, thus producing a photo-induced heating process of the entire nanostructure [4]. In fact, LSPR represents the way to transfer energy in such small nanoobjects: absorbed light induces unavoidable damping of the LSPR and generates a non-equilibrium electron distribution that decays via electron-electron collisions [5]. The hot electron gas re-establishes the equilibrium via lattice phonons that transfer generated energy to the surrounding medium, resulting in heat generation by the Joule effect. This heat generation increases the temperature inside the sample and brings the system out of equilibrium. The temperature of the environment is thus increased to reach a new equilibrium [4-5].

Since nano-localized temperature variation is the most important effect for applications ranging from nanomedicine to photonics, an important challenge in this research field is represented by the ability to estimate and measure the temperature variation at the surface of the NPs under optical illumination.

To explore the process of photo-induced heating, we consider a spherical metallic NP surrounded by air and illuminated by a monochromatic light characterized by an intensity of the external electric field amplitude $E_{0} \approx 10 \mathrm{~V} / \mathrm{m}$ and a wavelength $\lambda=532 \mathrm{~nm}$, corresponding to the LSPR maximum frequency of an Au NP (Fig. 1, left). In this condition, in fact, a significant local enhancement of the electric field strength near the metal/dielectric interface occurs [6].

Simulations based on the finite element method are used to implement both geometrical and thermal models. In particular, a 2D simulation is built by considering a spherical nanoparticle characterized by a radius $R_{n p}=10 \mathrm{~nm}$ and surrounded by air in order to estimate the enhancement of the electric field around the NP and the consequent temperature variation.

Due to strong resonance, the near-field around the NP is greatly enhanced with respect to the incident field $E_{0}$ at the frequency corresponding to the LSPR. In the dipolar limit, the electric field $E$ at the surface of a metal NP is given by [7]:

$$
E_{\text {surface }}=\frac{(1+k) \varepsilon_{m}}{\left(\varepsilon+k \varepsilon_{m}\right)} E_{0}
$$

where $\varepsilon$ is the dielectric function of the metal, $\varepsilon_{m}$ is the dielectric constant of the surrounding medium, $k$ is the shape factor that incorporates the dependence of the polarizability on the geometry of the surface that defines the electron oscillations (for a sphere $k=2$ ) [8-9]. As we can see from Fig. 1 (on the right) the maximum of the electric field around the NP reaches the value $2.710^{2} \mathrm{~V} / \mathrm{m}$.

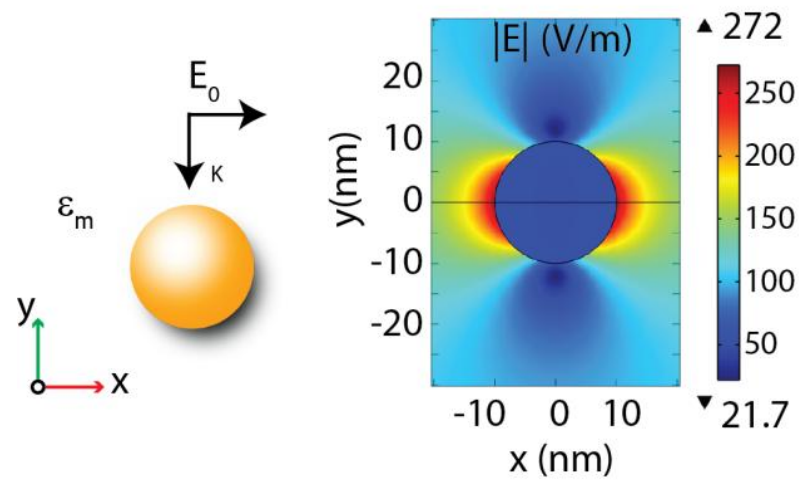

Fig. 1. Left: Sketch of the system. Right: Map of the electric field around the NP.

Once the enhancement of the electric field around the NP is estimated, in order to obtain the corresponding temperature increase with respect to the environment temperature $\left(T-T_{0}\right)$, we solve the equation governing purely conductive heat transfer:

$$
\rho C_{p} \frac{\partial T}{\partial t}+\nabla \cdot(-k \nabla T)=Q
$$

where: $\rho$ is the volumetric mass density (SI unit: $\mathrm{kg} / \mathrm{m}^{3}$ ), $C_{p}$ is the specific heat capacity at constant pressure (SI unit: $\mathrm{J} /(\mathrm{kg} \cdot \mathrm{K})), T$ is the absolute temperature (SI unit: $\mathrm{K}$ ), 
$k$ is the thermal conductivity (SI unit: $\mathrm{W} /(\mathrm{m} \cdot \mathrm{K})), Q$ is the heat sources density (SI unit: $\mathrm{W} / \mathrm{m}^{3}$ ). In this way, we can obtain a map of the temperature increase $\left(T-T_{0}\right)$ given by the Coulomb potential outside the NP [10] (Fig. 2, left). The dimensional analysis of Eq. 2 leads to the estimation of a typical time scale required to reach the steady-state regime, which in our system is reached in about $0.1 \mu \mathrm{s}$ (see Fig. 2, right). The maximum temperature variation obtained for a single NP, after this time, turns to be equal to $\left(1.04 \times 10^{-6}\right){ }^{\circ} \mathrm{K}$.
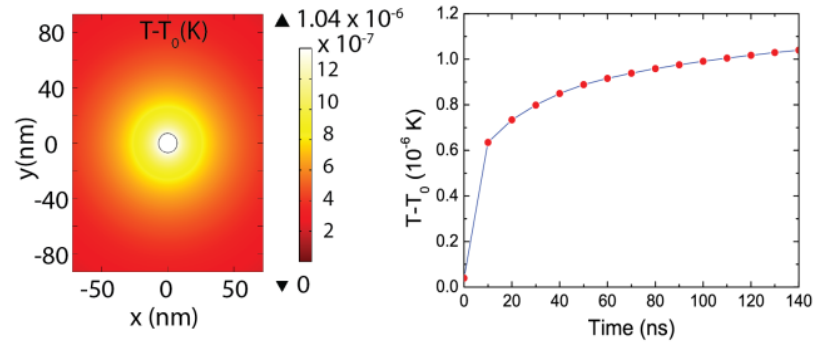

Fig. 2. Left: Map of temperature variation around the Au NP. Right: Temperature variation around the Au NP surface as a function of time, in the range $10-140 \mathrm{~ns}$

As reported in literature, this thermal effect is strongly related to the plasmonic coupling between GNPs [10-11]. Thus, in order to evaluate the minimum distance at which the thermal effect related to adjacent NPs can be considered as addictive, we place a second nanoparticle in the system, at a certain distance $d$ (edge-to-edge) from the first one (Fig. 3, inset).

It can be argued that, when the two metal NPs are brought close to each other, a near-field interaction takes place due to the overlap of the plasmon oscillations of the two NPs [12-13]. In this case, the electric field $E$ acting on each particle can be written as the sum of the incident light field $E_{0}$ and the near-field $E_{n f}$ that is due to the neighboring particle:

$$
E=E_{0}+E_{n f}
$$

In order to quantify the contribution of this coupling effect, finite element method based simulations of the system have been performed and the results are reported in Fig. 3. A strong enhancement of the electric field, estimated on the surface of one of the two NPs, is observed when the inter-particle gap is approximately less than ten particle radii $\left(R_{n p}\right)$.

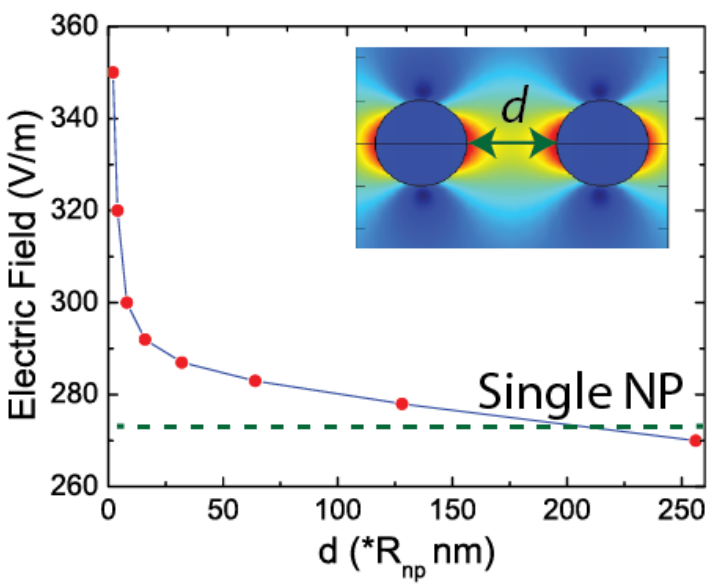

Fig. 3. Electric field estimated on the surface of an Au NP as a function of the distance with a second NPs.

The calculated maximum amplitude of the field is about $3.5 \times 10^{2} \mathrm{~V} / \mathrm{m}$, corresponding to the edge-to-edge distance $d=2 R_{n p}$. The field decreases to the single particle value $\left(2.7 \times 10^{2} \mathrm{~V} / \mathrm{m}\right.$, Fig. 1) when the gap is increased to $d \geq 250 R_{n p}$ and the NPs can be considered uncoupled.

This trend has a strong impact on the temperature variation, estimated on the Au NP surface, showing behavior very close to that observed for the electric field.

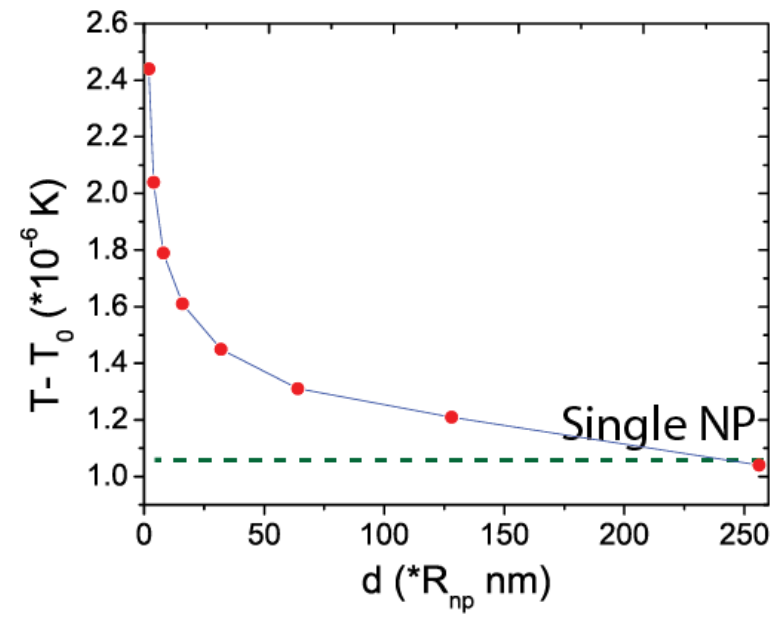

Fig. 4. Temperature variation estimated on the surface of an Au NP as a function of the inter-particle gap $d$ (edge-to-edge). The distance $d$ is calculated as a multiple of the radius $R_{n p}$ of the Au NP.

Indeed, as reported in Fig. 4, by exceeding the $d=50 R_{n p}$ distance, we find again the same temperature variation characteristics observed for a single NP $\left(1.04 \times 10^{-6} \mathrm{~K}\right)$. This suggests that, in a collection of NPs, if the average inter-distance is quite large, we can find a condition of spatially localized temperature in which the thermal effect due to each NP can be considered as "isolated" despite NPs being part of a collection. 
The analysis presented here shows how the distance between two NPs plays a key role in the confinement of light induced temperature variations at the nanoscale. In a collection of metallic NPs, the thermal response of a single plasmonic unit, in fact, strongly depends on the plasmonic coupling with close NPs. The substantial degree of control achieved through simulations over the temperature profile represents a useful tool to develop a model to investigate both localized and non-localized opto-thermal effects.

The research leading to these results has received support and funding from the Italian project "Nano-Lase"-PRIN 2012, Protocol No. 2012JHFYMC. The authors would like to acknowledge networking support by the COST Action IC1208.

\section{References}

[1] E. Hutter, J.H. Fendler, Advanced Materials 16, 1685 (2004) http://onlinelibrary.wiley.com/doi/10.1002/adma.200400271/abstract.

[2] L.M. Liz-Marzán, C.J. Murphy, J. Wang, Chemical Society Reviews, 43(11), 3820 (2014). http://pubs.rsc.org/en/content/articlelanding/ 2014/cs/c4cs90026j\#!divAbstract

[3] S.A. Maier, Plasmonics: fundamentals and applications (Springer Science \& Business Media, 2007), http://www.springer.com/ us/book/9780387331508

[4] B. Palpant, Photothermal properties of gold nanoparticles. Gold nanoparticles in physics, chemistry and biology (Imperial College Press, London, 2012).

[5] G. Baffou, R. Quidant, Laser Photon. Rev. 7(2), 171 (2013). http://onlinelibrary.wiley.com/doi/10.1002/lpor.201200003/abstract.

[6] M. Pelton, J. Aizpurua, G. Bryant, Laser Photon. Rev. 2(3), 136 (2008).

[7] U. Kreibig, M. Vollmer, Optical properties of metal clusters (Springer Science \& Business Media, 2013). http://www.springer.com/us/book/ 9783540578369.

[8] K. Prashant, S. Eustis, M.A. El-Sayed, J. Phys. Chem. B 110(37), 18243 (2006). http://pubs.acs.org/doi/abs/10.1021/jp063879z.

[9] P.K. Jain, M.A. El-Sayed, J. Phys. Chem. C, 112(13), 4954 (2008) http://pubs.acs.org/doi/abs/10.1021/jp7120356.

[10] G. Baffou, R. Quidant, F.J. García de Abajo, ACS Nano, 4(2), 709 (2010). http://pubs.acs.org/doi/abs/10.1021/nn901144d

[11] P.O. Chapuis, M. Laroche, S. Volz, J.J. Greffet, App. Phys. Lett. 92(20), 201906 (2008). http://aip.scitation.org/doi/full/10.1063/ 1.2931062

[12] P.K. Jain, M.A. El-Sayed, Chem. Phys. Lett. 487(4), 153 (2010). http://www.sciencedirect.com/science/article/pii/S000926141000119

[13] U. Cataldi, R. Caputo, Y. Kurylyak, G. Klein, M. Chekini, C. Umeton, T. Bürgi, J. Mater. Chem. C 2, 7927 (2014). http://pubs.rsc.org/en/ Content/ArticleLanding/2014/TC/c4tc01607f\#!divAbstract 